\title{
Optimized State Space Grids for Abstractions
}

\author{
Alexander Weber, Matthias Rungger and Gunther Reissig
}

\begin{abstract}
The practical impact of abstraction-based controller synthesis methods is currently limited by the immense computational effort for obtaining abstractions. In this note we focus on a recently proposed method to compute abstractions whose state space is a cover of the state space of the plant by congruent hyper-intervals. The problem of how to choose the size of the hyper-intervals so as to obtain computable and useful abstractions is unsolved. This note provides a twofold contribution towards a solution. Firstly, we present a functional to predict the computational effort for the abstraction to be computed. Secondly, we propose a method for choosing the aspect ratio of the hyper-intervals when their volume is fixed. More precisely, we propose to choose the aspect ratio so as to minimize a predicted number of transitions of the abstraction to be computed, in order to reduce the computational effort. To this end, we derive a functional to predict the number of transitions in dependence of the aspect ratio. The functional is to be minimized subject to suitable constraints. We characterize the unique solvability of the respective optimization problem and prove that it transforms, under appropriate assumptions, into an equivalent convex problem with strictly convex objective. The latter problem can then be globally solved using standard numerical methods. We demonstrate our approach on an example.
\end{abstract}

\section{Index Terms}

Discrete abstraction, symbolic control, automated synthesis, Djoković-London functional; MSC: Primary, 93B51; Secondary, 93B52, 93C10, 93C30, 93C55, 93C57, 93C65

\section{INTRODUCTION}

The concept of abstraction-based controller synthesis is a fully automated procedure to design feedback controllers that enforce predefined, possibly complex, specifications on nonlinear control systems [1], [2]. The procedure comprises three steps [2]. The first step is to transfer the actual control system ("plant") together with the predefined specification to an auxiliary control system, known as abstraction or symbolic model, and an auxiliary specification. In the second step, the auxiliary control problem is solved. The last step is to refine the obtained controller ("abstract controller") to a controller for the actual control problem. The practical impact of the approach is currently limited by the immense computational effort for the first step, i.e., for obtaining abstractions.

Various methods to reduce the computational effort of this procedure exist in literature, e.g. [3][10]. The methods in [3]-[6] merge the first and second step in previous scheme in order to compute the abstraction only partially. The methods in [6]-[8] locally refine symbolic models to reduce the number of required abstract states. In [9], [10] the state space of the plant is not discretized but finite 'sequences of inputs are used as abstract states.

This paper is the first to establish a reduction method for abstractions that are based on feedback refinement relations [1], [11]. Moreover, for the first time, a functional is presented that predicts the required computational resources for the abstraction to be computed.

Abstractions based on feedback refinement relations can be constructed for plants whose dynamics are governed by nonlinear differential equations subject to perturbations. Moreover, in contrast to

G. Reissig and A. Weber are with the University of the Federal Armed Forces Munich, Dept. Aerospace Eng., Chair of Control Eng. (LRT-15), D-85577 Neubiberg (Munich), Germany, http://www.reiszig.de/gunther/, a.weber@unibw.de

M. Rungger is with the Hybrid Control Systems Group at the Department of Electrical and Computer Engineering at the Technical University of Munich, Germany, matthias.rungger@tum.de

This work has been supported by the German Research Foundation (DFG) under grants no. RE 1249/3-2 and RE 1249/4-1. This is the accepted version of a paper published in IEEE Trans. Automat. Control, vol. 62, no. 11, pp. 5816-5821, 2017, DOI:10.1109/TAC.2016.2642794. 
other system relations, the induced controllers for the actual control problem merely consist of the abstract controller and a static quantizer [1].

The scheme for computing such an abstraction is as follows. First, the $n$-dimensional real state space of the plant is discretized by means of a cover to obtain the states of the abstraction, where the vast majority of the elements of the cover are translated copies of the hyper-interval

$$
\left[-\frac{\eta_{1}}{2}, \frac{\eta_{1}}{2}\right] \times \ldots \times\left[-\frac{\eta_{n}}{2}, \frac{\eta_{n}}{2}\right], \quad \eta_{1}, \ldots, \eta_{n}>0,
$$

which are aligned on a uniform grid. Second, attainable sets of the sets in the cover are overapproximated by hyper-intervals to obtain the transitions in the abstraction.

The goal of this work is to provide a heuristic for choosing $\eta_{1}, \ldots, \eta_{n}$ in (1) so as to reduce the memory and time consumption when computing abstractions for which the volume $\eta_{1} \cdot \eta_{2} \ldots \eta_{n}$ of (1) is predefined. The key idea here is the minimization of the expected number of transitions. As a first step towards this goal, we propose to use the functional

$$
E(\eta)=\prod_{i=1}^{n} \frac{1}{\eta_{i}}\left(p_{i}+\sum_{j=1}^{n} A_{i, j} \eta_{j}\right)
$$

to estimate the number of transitions per abstract state and input symbol in dependence of $\eta=$ $\left(\eta_{1}, \ldots, \eta_{n}\right)$ in (1). Here, the nonnegative $n \times n$-matrix $A$ and the $n$-dimensional nonnegative vector $p$ depend on the particular plant dynamics and on bounds on disturbances.

In the next step we study the minimization of (2) subject to a constraint that prescribes the volume of (1). For this, in general, non-convex optimization problem, we characterize the existence of a unique solution. To this end, we eliminate non-convexity by suitably transforming (2) and show that under appropriate assumptions the auxiliary optimization problem has strictly convex objective and can be globally solved by standard numerical methods. These results then allow us to establish the requested heuristic. We finally demonstrate our approach on an example.

Our results on the functional in (2) recover and extend previous results for the special case $p=0$, which plays a part in diagonal scaling of nonnegative matrices into doubly stochastic form [12], [13]. Some of our results have been announced in [14].

\section{Preliminaries}

1) Notation: $\mathbb{R}, \mathbb{R}_{+}$and $\mathbb{Z}$ denote the sets of real numbers, nonnegative real numbers, and integers, respectively. $[a, b],] a, b[,[a, b[$, and $] a, b]$ denote closed, open and half-open, respectively, intervals with end points $a$ and $b$. $[a ; b]], a ; b[,[a ; b[$, and $] a ; b]$ stand for discrete intervals, e.g. $[a ; b]=[a, b] \cap \mathbb{Z}$, $\left[1 ; 4\left[=\{1,2,3\}\right.\right.$, and $\left[0 ; 0\left[=\emptyset\right.\right.$. In $\mathbb{R}^{n}$, the relations $<, \leq, \geq,>$ are defined component-wise, e.g., $a<b$ iff $a_{i}<b_{i}$ for all $i \in[1 ; n]$. For $x \in \mathbb{R}^{n}$ we define $|x|=\left(\left|x_{1}\right|, \ldots,\left|x_{n}\right|\right)$. For $a, b \in(\mathbb{R} \cup\{\infty,-\infty\})^{n}, a \leq b$, the closed hyper-interval $\llbracket a, b \rrbracket$ is defined by $\llbracket a, b \rrbracket=\mathbb{R}^{n} \cap\left(\left[a_{1}, b_{1}\right] \times \cdots \times\left[a_{n}, b_{n}\right]\right) .\langle\cdot \mid \cdot\rangle$ stands for the standard Euclidean inner product, i.e., $\langle x \mid y\rangle=\sum_{i=1}^{n} x_{i} y_{i} \cdot\|\cdot\|_{p}$ stands for the usual $p$-norm, $p \in[1, \infty]$.

$f: A \rightrightarrows B$ denotes a set-valued map of $A$ into $B$, whereas $f: A \rightarrow B$ denotes an ordinary map; see [15]. If $f$ is set-valued, then $f$ is strict if $f(a) \neq \emptyset$ for every $a$. We identify set-valued maps $f: A \rightrightarrows B$ with binary relations on $A \times B$, i.e., $(a, b) \in f$ iff $b \in f(a)$. $f \circ g$ denotes the composition of $f$ and $g$, $(f \circ g)(x)=f(g(x))$.

We denote the vector $(1, \ldots, 1) \in \mathbb{R}^{k}$ by $\mathbf{1}$ and the identity map $X \rightarrow X: x \mapsto x$ by id. The dimension $k$ and the domain of definition $X$ respectively will always be clear from the context. A cover of a set $X$ is a set of subsets of $X$ whose union equals $X$.

2) nonnegative matrices: A matrix $A$ is nonnegative if $A \in \mathbb{R}_{+}^{n \times n}$ and it is essentially nonnegative if $A \in \mathbb{R}^{n \times n}$ and $A_{i, j} \geq 0$ whenever $i \neq j$. A matrix $A \in \mathbb{R}^{n \times n}$ is irreducible if for any $r, s \in[1 ; n]$, $r \neq s$ there exist distinct indices $i_{1}, \ldots, i_{m} \in[1 ; n]$ satisfying $i_{1}=r, i_{m}=s$ and $A_{i_{k}, i_{k+1}}>0$ for all $k \in[1 ; m[$. Otherwise, $A$ is reducible. 


\section{Computation OF ABSTRACTiOnS}

This section gives a brief exposition of the method to compute abstractions from [1]. We consider control systems governed by nonlinear differential inclusions of the form

$$
\dot{\xi}(t) \in f(\xi(t), u)+\llbracket-w, w \rrbracket,
$$

where $f: \mathbb{R}^{n} \times \bar{U} \rightarrow \mathbb{R}^{n}, \bar{U} \subseteq \mathbb{R}^{m}$ is nonempty, $f(\cdot, u)$ is locally Lipschitz for all $u \in \bar{U}$, and $w \in \mathbb{R}_{+}^{n}$ is a component-wise bound on perturbations to the dynamics of the control system. For $\tau>0$ a solution of (3) on $[0, \tau]$ with (constant) input $u \in \bar{U}$ is an absolutely continuous function $\xi:[0, \tau] \rightarrow \mathbb{R}^{n}$ that fulfills (3) for almost every $t \in[0, \tau][16]$.

We formalize sampled versions of control systems (3) in a notion of system as given below.

III.1 Definition. A system is a triple $(X, U, F)$, where $X$ and $U$ are nonempty sets and $F: X \times U \rightrightarrows$ $X$.

We call the sets $X$ and $U$ the state and input alphabet, respectively. The map $F$ is called the transition function.

III.2 Definition. Let $S=(X, U, F)$ be a system and $\tau>0$. We say that $S$ is the sampled system associated with the control system (3) and the sampling time $\tau$, if $X=\mathbb{R}^{n}, U=\bar{U}$ and the following holds: $x_{1} \in F\left(x_{0}, u\right)$ iff there exists a solution $\xi$ of (3) on $[0, \tau]$ with input $u$ so that $\xi(0)=x_{0}$ and $\xi(\tau)=x_{1}$.

We relate two systems to each other by feedback refinement relations. We introduce this concept as follows.

For a system $S=(X, U, F)$ and $x \in X$ let

$$
U_{S}(x)=\{u \in U \mid F(x, u) \neq \emptyset\} .
$$

III.3 Definition. Let $S_{i}=\left(X_{i}, U_{i}, F_{i}\right), i \in\{1,2\}$ be two systems such that $U_{2} \subseteq U_{1}$. A feedback refinement relation from $S_{1}$ to $S_{2}$ is a strict relation $Q \subseteq X_{1} \times X_{2}$ satisfying

(i) $U_{S_{2}}\left(x_{2}\right) \subseteq U_{S_{1}}\left(x_{1}\right)$,

(ii) $u \in U_{S_{2}}\left(x_{2}\right) \Longrightarrow Q\left(F_{1}\left(x_{1}, u\right)\right) \subseteq F_{2}\left(x_{2}, u\right)$

for all $\left(x_{1}, x_{2}\right) \in Q$.

We write $S_{1} \preccurlyeq Q S_{2}$ if $Q$ is a feedback refinement relation from $S_{1}$ to $S_{2}$. If $S_{1} \preccurlyeq Q S_{2}$ we say that $S_{2}$ is an abstraction for $S_{1}$.

A feedback refinement relation $Q$ from $S_{1}$ to $S_{2}$ associates states of $S_{1}$ with states of $S_{2}$ and imposes conditions on the images of the transition functions at associated states. The relation $Q$ also serves as an interface to be added to the abstract controller in order to refine it into a controller for the actual plant. We refer the reader to [1] for a formal definition of the closed loop, the details of the synthesis procedure, and in particular, for a proof of the fact that the refined controller actually solves the control problem for the plant $S_{1}$. The framework also allows for bounded measurement errors $P: \mathbb{R}^{n} \rightrightarrows \mathbb{R}^{n}$ of the form

$$
P(x)=x+\llbracket-z, z \rrbracket
$$

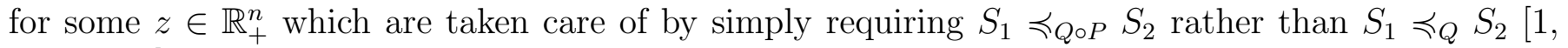
Sec. VI.B].

In what follows, we discuss the computation of abstractions $S_{2}=\left(X_{2}, F_{2}, U_{2}\right)$ satisfying $S_{1} \preccurlyeq Q_{\circ P} S_{2}$, for a sampled system $S_{1}$ associated with (3), where we restrict our attention to abstractions whose state alphabet $X_{2}$ is a cover of the state alphabet of $S_{1}$. The elements of $X_{2}$ are nonempty, closed hyper-intervals, which we call cells. We divide $X_{2}$ into two subsets, which we interpret as "real" 
quantizer symbols and overflow symbols, respectively. See [17, Sec. III.A]. We let the former subset, subsequently denoted by $\bar{X}_{2}$, consist of congruent cells that are aligned on the uniform grid

$$
\eta \mathbb{Z}^{n}=\left\{c \in \mathbb{R}^{n} \mid \exists_{k \in \mathbb{Z}^{n}} \forall_{i \in[1 ; n]} c_{i}=k_{i} \eta_{i}\right\}
$$

with grid parameter $\eta \in\left(\mathbb{R}_{+} \backslash\{0\}\right)^{n}$, i.e.,

$$
x_{2} \in \bar{X}_{2} \Rightarrow \exists_{c \in \eta \mathbb{Z}^{n}} x_{2}=c+\llbracket-\eta / 2, \eta / 2 \rrbracket .
$$

The computation of the map $F_{2}$ on $\bar{X}_{2} \times U_{2}$ will be based on overapproximating the attainable sets of the cells in $\bar{X}_{2}$ under the flow of (3). For that purpose, we will define growth bounds below. Growth bounds have been introduced in [1, Sec. VIII.A], where their important features are also discussed.

We denote by $\varphi$ the general solution of the unperturbed control system associated with (3). More formally, if $x_{0} \in \mathbb{R}^{n}, u \in \bar{U}$, then $\varphi\left(t, x_{0}, u\right)$ is the value at time $t$ of the solution of the initial value problem $\dot{x}=f(x, u), x(0)=x_{0}$.

III.4 Definition. Let $\tau>0, K \subseteq \mathbb{R}^{n}$, and $\bar{U}^{\prime} \subseteq \bar{U} . A$ map $\beta: \mathbb{R}_{+}^{n} \times \bar{U}^{\prime} \rightarrow \mathbb{R}_{+}^{n}$ is a growth bound on $K, \bar{U}^{\prime}$ associated with $\tau$ and (3) if

(i) $\beta(r, u) \geq \beta\left(r^{\prime}, u\right)$ whenever $r \geq r^{\prime}$ and $u \in \bar{U}^{\prime}$,

(ii) $[0, \tau] \times \bar{K} \times \bar{U}^{\prime} \subseteq \operatorname{dom} \varphi$ and if $\xi$ is a solution of (3) on $[0, \tau]$ with input $u \in \bar{U}^{\prime}$ and $\xi(0), p \in K$ then $|\xi(\tau)-\varphi(\tau, p, u)| \leq \beta(|\xi(0)-p|, u)$.

Explicit growth bounds of the form

$$
\beta(r, u)=\mathrm{e}^{L(u) \tau} r+v(u)
$$

where $v(u) \in \mathbb{R}_{+}^{n}$ and the matrix $L(u) \in \mathbb{R}^{n \times n}$ is essentially nonnegative, can be computed under mild assumptions [1]. The next result, which extends [1, Th. VIII.4] to the case of multiple growth bounds, is the key to the computation of abstractions.

III.5 Theorem. Let $S_{1}=\left(X_{1}, U_{1}, F_{1}\right)$ be the sampled system associated with (3) and sampling time $\tau>0$, and let $P$ be given by (4). Let $S_{2}=\left(X_{2}, U_{2}, F_{2}\right)$ be a system, where $X_{2}$ is a cover of $X_{1}$ by nonempty, closed hyper-intervals and $U_{2} \subseteq U_{1}$. Consider a subset $\bar{X}_{2} \subseteq X_{2}$ that satisfies (6), and for any $x_{2} \in \bar{X}_{2}$ let $\beta_{x_{2}}$ be a growth bound on $P\left(x_{2}\right), U_{2}$ associated with $\tau$ and (3). Suppose that $F_{2}$ is given by

(i) $F_{2}\left(x_{2}, u\right)=\emptyset$ whenever $x_{2} \in X_{2} \backslash \bar{X}_{2}, u \in U_{2}$, and

(ii) for $x_{2} \in \bar{X}_{2}, x_{2}^{\prime} \in X_{2}$ and $u \in U_{2}$ we have

$$
x_{2}^{\prime} \in F_{2}\left(x_{2}, u\right) \Longleftrightarrow\left(c+\llbracket-r^{\prime}, r^{\prime} \rrbracket\right) \cap P\left(x_{2}^{\prime}\right) \neq \emptyset,
$$

with $r^{\prime}=\beta_{x_{2}}(\eta / 2+z, u), x_{2}=\bar{c}+\llbracket-\eta / 2, \eta / 2 \rrbracket$, and

$$
c=\varphi(\tau, \bar{c}, u) .
$$

Then we have $S_{1} \preccurlyeq{ }_{Q \circ P} S_{2}$, with $Q \subseteq X_{1} \times X_{2}$ defined by $\left(x_{1}, x_{2}\right) \in Q$ iff $x_{1} \in x_{2}$.

Theorem III.5 leads to constructive means to compute abstractions basically as follows. For every cell $x_{2}=\bar{c}+\llbracket-\eta / 2, \eta / 2 \rrbracket \in \bar{X}_{2}$ and input symbol $u \in U_{2}$

1) compute $c=\varphi(\tau, \bar{c}, u)$ and $r^{\prime}=\beta_{x_{2}}(\eta / 2+z, u)$,

2) determine all cells $c^{\prime}+\llbracket-\eta / 2, \eta / 2 \rrbracket \in X_{2}$ that satisfy

$$
\left(c+\llbracket-r^{\prime}, r^{\prime} \rrbracket\right) \cap\left(c^{\prime}+\llbracket-\eta / 2-z, \eta / 2+z \rrbracket\right) \neq \emptyset,
$$

and define $F_{2}\left(x_{2}, u\right)$ as the set of all such cells.

Proof of Theorem III.5. We have to verify (i),(ii) in Definition III.3 with $Q \circ P$ in place of $Q$. To see (i) let $\left(x_{1}, x_{2}\right) \in Q \circ P$ and $u \in U_{S_{2}}\left(x_{2}\right)$. Then, $F_{2}\left(x_{2}, u\right) \neq \emptyset$ and $x_{2} \in \bar{X}_{2}$ by the assumptions on $u$ and $F_{2}$. By our assumption on the growth bound $\beta_{x_{2}}$ it follows $F_{1}\left(x_{1}, u\right) \neq \emptyset$, thus $u \in U_{S_{1}}\left(x_{1}\right)$. 
To see (ii) in Definition III.3, let $\left(x_{1}, x_{2}\right) \in Q \circ P, u \in U_{S_{2}}\left(x_{2}\right)$ and $x_{2}^{\prime} \in(Q \circ P)\left(F_{1}\left(x_{1}, u\right)\right)$. It follows that $F_{1}\left(x_{1}, u\right) \cap P\left(x_{2}^{\prime}\right) \neq \emptyset$. Indeed, $x_{2}^{\prime} \in(Q \circ P)\left(x_{1}^{\prime}\right)$ for some $x_{1}^{\prime} \in F_{1}\left(x_{1}, u\right)$, thus $\left(x_{1}^{\prime}, P\left(x_{2}^{\prime}\right)\right) \in Q$, so $x_{1}^{\prime} \in P\left(x_{2}^{\prime}\right)$. Next, from $x_{1} \in P\left(x_{2}\right)$ and the properties of $\beta_{x_{2}}$ it follows that $F_{1}\left(x_{1}, u\right) \subseteq \varphi(\tau, \bar{c}, u)+$ $\llbracket-r^{\prime}, r^{\prime} \rrbracket$, where $x_{2}=\bar{c}+\llbracket-\eta / 2, \eta / 2 \rrbracket$ and $r^{\prime}=\beta_{x_{2}}(\eta / 2+z, u)$. Thus, $\left(\varphi(\tau, \bar{c}, u)+\llbracket-r^{\prime}, r^{\prime} \rrbracket\right) \cap P\left(x_{2}^{\prime}\right) \neq \emptyset$, and by the properties of $F_{2}$, we conclude $x_{2}^{\prime} \in F_{2}\left(x_{2}, u\right)$.

\section{Estimation of the Size of ABStractions}

The size of an abstraction $S_{2}=\left(X_{2}, U_{2}, F_{2}\right)$ that is obtained by Theorem III.5 is given by the number of transitions. To obtain a prediction on this size, we will disregard overflow symbols by assuming $\bar{X}_{2}=X_{2}$, and in addition, we will assume $c$ in (8) is a random vector uniformly distributed on the cells. Then, the following theorem shows that the function $E:\left(\mathbb{R}_{+} \backslash\{0\}\right)^{n} \rightarrow \mathbb{R}_{+}$given by (2) with $A \in \mathbb{R}_{+}^{n \times n}, p \in \mathbb{R}_{+}^{n}$ provides a prediction on the cardinality of $F_{2}\left(x_{2}, u\right)$ for fixed $\left(x_{2}, u\right) \in \bar{X}_{2} \times U_{2}$ in dependence of the grid parameter $\eta$.

The key property of the functional $E$ is that it also provides an accurate prediction when actually computing abstractions. (See Section VI.)

IV.1 Theorem. Assume the hypotheses of Theorem III.5 with $\bar{X}_{2}=X_{2}$. Let $\left(x_{2}, u\right) \in X_{2} \times U_{2}$ and let $\beta:=\beta_{x_{2}}$ in Theorem III.5 be of the form ( 7$)$, where $v(u) \geq 0$ and $L(u)$ is essentially nonnegative. For $c$ in (8) assume in place of (9) that $c$ is an $n$-dimensional vector of independent random variables $c_{i}, i \in[1 ; n]$ each of which is uniformly distributed on some interval of length $\eta_{i}$. Then the expected value of the number of cells in $F_{2}\left(x_{2}, u\right)$ is given by $E(\eta)$ in (2) with

$$
A=\mathrm{id}+\mathrm{e}^{L(u) \tau} \quad \text { and } \quad p=2(A z+v(u)) .
$$

Proof. The number of elements in $F_{2}(x, u)$ for $x=\bar{c}+\llbracket-\eta / 2, \eta / 2 \rrbracket$ is given by

$$
N_{\eta}^{n}\left(c, r^{\prime}\right)=\left|\left\{p \in \eta \mathbb{Z}^{n} \mid p \in c+\llbracket-r^{\prime}, r^{\prime} \rrbracket\right\}\right|
$$

with the random vector $c$ and $r^{\prime}=r+\mathrm{e}^{L(u) \tau} r+v(u)$ where $r=\eta / 2+z$. Here, $|X|$ stands for the cardinality of the set $X$. We have $2 r^{\prime}=2 r+2\left(\mathrm{e}^{L(u) \tau} r+v(u)\right)=p+A \eta$. The proof is therefore completed by the next lemma.

IV.2 Lemma. Consider the grid $\eta \mathbb{Z}^{n}$ in (5) with $\eta \in \mathbb{R}^{n}, \eta>0$. Let $r \in \mathbb{R}_{+}^{n}$ and let $c_{i}, i \in[1 ; n]$ be $n$ independent random variables, where each $c_{i}$ is uniformly distributed on some interval of length $\eta_{i}$. Then the expected value of the number $N_{\eta}^{n}(c, r)$ defined in (11) is given by $\prod_{i=1}^{n} 2 r_{i} / \eta_{i}$.

Proof. Note that $N_{\eta}^{n}(c, r)=\prod_{i=1}^{n} N_{\eta_{i}}^{1}\left(c_{i}, r_{i}\right)$ and since the $c_{i}$ are mutually independent, the expected value of $N_{\eta}^{n}(c, r)$ is given as the product of the expected values of $N_{\eta_{i}}^{1}\left(c_{i}, r_{i}\right)$. Moreover, $N_{\eta_{i}}^{1}\left(c_{i}, r_{i}\right)=$ $N_{1}^{1}\left(c_{i} / \eta_{i}, r_{i} / \eta_{i}\right)$ and $N_{1}^{1}(x+1, \hat{\eta})=N_{1}^{1}(x, \hat{\eta})$ for every $x \in \mathbb{R}$ and $\hat{\eta} \in \mathbb{R}_{+}$. Hence, it suffices to consider $N_{1}^{1}(\hat{c}, \hat{\eta})$ with $\hat{c}$ being uniformly distributed on $[0,1]$ and $\hat{\eta} \in \mathbb{R}_{+}$. The expected value of $N_{1}^{1}(\hat{c}, \hat{\eta})$ is given by $2 \hat{\eta}$. Indeed, if $\hat{\eta}=k+\varepsilon$ with $k \in \mathbb{Z}_{+}$and $\varepsilon \in[0,1 / 2[$, then we obtain

$$
\int_{0}^{1} N_{1}^{1}(x, \hat{\eta}) \mathrm{d} x=(2 k+1) \varepsilon+2 k(1-2 \varepsilon)+(2 k+1) \varepsilon=2 \hat{\eta}
$$

by separating the integration interval into $[0, \varepsilon],[\varepsilon, 1-\varepsilon]$ and $[1-\varepsilon, 1]$. The case $\varepsilon \in[1 / 2,1[$ is similar.

\section{Minimization of the size of abstractions}

Theorem IV.1 motivates the following on the computation of abstractions in the special case that the growth bounds in Theorem III.5 coincide and do not depend on the input symbol, i.e., $\beta_{x_{2}}(r, u)=$ $\beta_{x_{2}^{\prime}}\left(r, u^{\prime}\right)$ for any $\left(x_{2}, u\right),\left(x_{2}^{\prime}, u^{\prime}\right) \in \bar{X}_{2} \times U_{2}$, and any $r \in \mathbb{R}_{+}^{n}$ : Consider the abstractions for $S_{1}$ that have cells of volume $\exp (\gamma), \gamma \in \mathbb{R}$, and input alphabet $U_{2}$. Among those abstractions, the abstraction 
with the least expected size has cells that are aligned according grid parameter $\eta$, where $\eta$ is a solution of the optimization problem

$$
\min _{\xi>0} E(\xi) \text { subject to } \exp (\gamma)=\prod_{i=1}^{n} \xi_{i} \text {. }
$$

Unfortunately, the optimization problem (12) is non-convex if $n \geq 2$, and non-convex problems are notoriously difficult to solve.

The main results of this work, which are presented in this section, include a characterization of existence and uniqueness of $\eta$, and the means to numerically compute $\eta$, so that the just motivated heuristic to reduce the computational effort becomes applicable. We will also investigate the generalization of (12) to the case of arbitrary growth bounds.

To establish aforementioned characterization we first bypass non-convexity. To this end, consider a transformation of (12):

$$
\min _{x} g(x) \text { subject to } x \in V_{\gamma},
$$

where $g(x)=E(\exp (x))$ and

$$
V_{s}=\left\{v \in \mathbb{R}^{n} \mid v_{1}+\ldots+v_{n}=s\right\}
$$

for $s \in \mathbb{R}$. Here and subsequently, the exponential exp is taken component-wise whenever the argument is a vector. The result below lists the outstanding properties of $g$.

V.1 Theorem. Let $n \geq 2, A \in \mathbb{R}_{+}^{n \times n}, p \in \mathbb{R}_{+}^{n}$ and $\gamma \in \mathbb{R}$, and let $E, g$ and $V$ be defined as in (2) and above. Then $g$ is convex. Moreover, if all diagonal entries of $A$ are positive and $A$ or $\left(\begin{array}{ll}A & p \\ 1 & 1\end{array}\right)$ is irreducible then the assertions below hold.

(i) $g$ is strictly convex on $V_{\gamma}$. To be more precise, $g^{\prime \prime}(x) h^{2}>0$ for all $x \in V_{\gamma}$ and $h \in V_{0} \backslash\{0\}$.

(ii) Let $\mu$ be the smallest nonzero entry of $A$ and $p, c=(n-1)^{-1}$. Then $x \in V_{\gamma}$ implies

$$
g(x) \geq \mu^{n} \exp (-|\gamma| c) \exp \left(c\|x\|_{\infty}\right) .
$$

The above result implies that the optimization problem (13) is convex. Moreover, since (15) implies $g(x) \rightarrow \infty$ as $\|x\|_{\infty} \rightarrow \infty, x \in V_{\gamma}$, the problem (13) has a unique solution under the hypotheses of Theorem V.1 [18, 4.3.3], and thus, so has (12). Our result also shows that standard numerical methods will converge globally when applied to (13), e.g. [18, Sec. 14.5], and some will do so even if (13) is supplemented with a finite number of constraints of the form $a_{i} \leq x_{i}$ or $x_{i} \leq a_{i}, a_{i} \in \mathbb{R}[19$, Th. 1]. For completeness, we remark that global convergence can also be ensured if $g$ is strongly convex on $V_{\gamma}$, a property established in [14, Th. 3] for irreducible $A$. However, the property is not implied under the rather mild hypotheses of Theorem V.1, which are satisfied, in particular, if every component of the state is subject to some measurement error, i.e., if $z>0$ in (10), regardless of the dynamics of the plant $S_{1}$ under investigation. Finally, we note that checking irreducibility (for $n \geq 2$ ) is equivalent to finding strongly connected components in directed graphs [21, Th. 2.2.7], so irreducibility can be checked with linear time algorithms [20].

Proof of Theorem V.1. Convexity of $g$ has been established in [14, Th. 3]. We first prove (i). Define $R_{i}(x)=p_{i}+\sum_{j=1}^{n} A_{i, j} \mathrm{e}^{x_{j}}$ and $G(x)=\sum_{i=1}^{n} \ln R_{i}(x)$ to see that

$$
g(x)=\mathrm{e}^{-\gamma} \prod_{i=1}^{n} R_{i}(x)=\exp (G(x)-\gamma)
$$

for every $x \in V_{\gamma}$. It follows that $g^{\prime \prime}(x) h^{2}=g(x)\left(G^{\prime}(x) h\right)^{2}+g(x) G^{\prime \prime}(x) h^{2}$ and that $\left(G^{\prime}(x) h\right)^{2}+G^{\prime \prime}(x) h^{2}$ equals

$$
\left(\sum_{i=1}^{n} \frac{R_{i}^{\prime}(x) h}{R_{i}(x)}\right)^{2}+\sum_{i=1}^{n} \frac{R_{i}^{\prime \prime}(x) h^{2} R_{i}(x)-\left(R_{i}^{\prime}(x) h\right)^{2}}{\left(R_{i}(x)\right)^{2}}
$$


for all $x \in V_{\gamma}$ and all $h \in V_{0}$. Define vectors $a^{(i)}, b^{(i)} \in \mathbb{R}^{n+1}$ by $b_{n+1}^{(i)}=p_{i}^{1 / 2}, a_{n+1}^{(i)}=0, b_{j}^{(i)}=\left(A_{i, j} \mathrm{e}^{x_{j}}\right)^{1 / 2}$ and $a_{j}^{(i)}=b_{j}^{(i)} h_{j}$ for every $j \leq n$. Then $b^{(i)}$ is not a zero vector for any $i \in[1 ; n]$. Use (16) to see that $g(x)^{-1} g^{\prime \prime}(x) h^{2}$ equals

$$
\left(\sum_{i=1}^{n} \frac{\left\langle a^{(i)} \mid b^{(i)}\right\rangle}{\left\|b^{(i)}\right\|_{2}^{2}}\right)^{2}+\sum_{i=1}^{n} \frac{\left\|a^{(i)}\right\|_{2}^{2} \cdot\left\|b^{(i)}\right\|_{2}^{2}-\left\langle a^{(i)} \mid b^{(i)}\right\rangle^{2}}{\left\|b^{(i)}\right\|_{2}^{4}} .
$$

Now assume $g^{\prime \prime}(x) h^{2}=0$ for some $h \in V_{0}$ and let us show that $h=0$. Indeed, we deduce from (17) and Cauchy's inequality that for all $i \in[1 ; n]$ there exists $\lambda_{i} \in \mathbb{R}$ such that $a^{(i)}=\lambda_{i} b^{(i)}$. This equation implies a) $\lambda_{i}=0$ whenever $p_{i}>0$, b) $\lambda_{i}=h_{i}$ for any $i$ as $A_{i, i}>0$, and therefore c) $h_{i}=h_{j}$ whenever $A_{i, j}>0$. Next, assume that $h$ has two nonzero components $h_{r}, h_{s}$ such that $h_{r} \neq h_{s}$. By the irreducibility of $A$ or $\left(\begin{array}{cc}A & p \\ 1 & 1\end{array}\right)$ there exist distinct indices $i_{1}, \ldots, i_{m} \in[1 ; n+1]$ such that $r=i_{1}, s=i_{m}$ and at least one of the following cases occurs:

1) $n+1 \notin\left\{i_{1}, \ldots, i_{m}\right\}$ and $A_{i_{k}, i_{k+1}}>0$ for all $k \leq m-1$,

2) $p_{r}>0$

3) $m \geq 3, p_{i_{m-1}}>0$ and $A_{i_{k}, i_{k+1}}>0$ for all $k \leq m-2$.

The remarks a), b), c) above will exclude each of the three cases. Indeed, the first case is impossible as it implies $h_{r}=h_{s}$. The second case implies $h_{r}=0$, so cannot occur either. For the same reason, the third case is impossible as $h_{i_{m-1}}=0$ and $h_{r}=h_{i_{m-1}}$. Consequently, the nonzero entries of $h$ coincide. However, as (17) vanishes, we conclude $0=\sum_{i=1}^{n} \lambda_{i}=\sum_{i=1}^{n} h_{i}$, and so $h=0$.

Now we prove (ii). We begin with deriving an inequality that we use in the second part of the proof. To this end, note first that by our assumptions the following property holds for all $r \in[1 ; n]$ and all $s \in[1 ; n]$, or for all $r \in[1 ; n]$ and $s=n+1$ : There exists a subset of indices $\left\{i_{1}, \ldots, i_{m}\right\} \subseteq[1 ; n+1]$ such that $(r, s)=\left(i_{1}, i_{m}\right)$ and $\tilde{A}_{i_{k}, i_{k+1}}>0$ for all $k \in\left[1 ; m\left[\right.\right.$, where $\tilde{A}=\left(\begin{array}{cc}A & p \\ 0 & 1\end{array}\right)$. Now fix $(r, s)$ satisfying previous condition with the subset of indices $P:=\left\{i_{1}, \ldots, i_{m}\right\}$. Let $x \in \mathbb{R}^{n}$ and set $x_{n+1}:=0$ to see that

$$
\begin{aligned}
& g(x) \geq \mathrm{e}^{-\gamma}\left(\prod_{\substack{j=1 \\
j \notin P \backslash\left\{i_{m}\right\}}}^{n} \tilde{A}_{j, j} \exp \left(x_{j}\right)\right) \prod_{k=1}^{m-1} \tilde{A}_{i_{k}, i_{k+1}} \exp \left(x_{i_{k+1}}\right) \\
& \geq \mu^{n}\left(\prod_{j \in P} \exp \left(-x_{j}\right)\right) \prod_{k=2}^{m} \exp \left(x_{i_{k}}\right)=\mu^{n} \exp \left(x_{s}-x_{r}\right) .
\end{aligned}
$$

For the second part of the proof let $q \in[1 ; n]$ such that $\|x\|_{\infty}=\left|x_{q}\right|$ and observe $x_{q}-\gamma=-\sum_{r=1, r \neq q}^{n} x_{r}$. So, for some $s \in\{q, n+1\}$ we have

$$
\prod_{\substack{r=1 \\ r \neq q}}^{n} \exp \left(x_{s}-x_{r}\right)=\exp \left((n-1) x_{s}\right) \exp \left(x_{q}-\gamma\right) .
$$

Therefore, $\left(\mu^{-n} g(x)\right)^{n-1}$ is not less than (19) by applying (18) to each factor of the product in (19). This implies (15) if $x_{q} \geq 0$. If $x_{q}<0$ and if (18) holds for $s=n+1$ then (15) follows obviously. If $x_{q}<0$ and if (18) holds for all $r, s \in[1 ; n]$ then take the inverse on both sides of (19) with $s=q$, and use (18) with $r$ and $q$ in place of $s$ and $r$, respectively, to see that $\left(\mu^{-n} g(x)\right)^{n-1} \geq \exp \left(\gamma-n x_{q}\right)$. So, the proof is easily completed.

The announced characterization related to (12) is as follows.

V.2 Theorem. Let $\gamma \in \mathbb{R}$ and $E$ be defined as in (2) with $A$ having positive diagonal. Then the optimization problem (12) has a unique minimum point iff $A$ or $\left(\begin{array}{ll}A & p \\ 1 & 1\end{array}\right)$ is irreducible.

The proof of above theorem requires the next lemma. Below, $A^{\top}$ denotes the transpose of a matrix A. 
V.3 Lemma. Let $n \geq 2$, let $A \in \mathbb{R}^{n \times n}, p \in \mathbb{R}_{+}^{n}$. Assume that both $A$ and $\tilde{A}=\left(\begin{array}{cc}A & p \\ 1 & 1\end{array}\right)$ are reducible. Then $\tilde{P} \tilde{A} \tilde{P}^{\top}$ equals

$$
\left(\begin{array}{lll}
X & 0 & 0 \\
Z & Y & \bar{p} \\
\mathbf{1} & \mathbf{1} & 1
\end{array}\right)
$$

where $\tilde{P}=\left(\begin{array}{cc}P & 0 \\ 0 & 1\end{array}\right) \in \mathbb{R}^{(n+1) \times(n+1)}, P \in \mathbb{R}^{n \times n}$ is a permutation matrix, $X \in \mathbb{R}^{n_{1} \times n_{1}}, Y \in \mathbb{R}^{n_{2} \times n_{2}}$, $Z \in \mathbb{R}^{n_{2} \times n_{1}}, n_{1}, n_{2} \in\left[1 ; n\left[, n_{1}+n_{2}=n\right.\right.$ and $\bar{p} \in \mathbb{R}_{+}^{n_{2}}$.

Proof. There exists a permutation matrix $T \in \mathbb{R}^{(n+1) \times(n+1)}$ such that $T \tilde{A} T^{\top}=\left(\begin{array}{ll}B & 0 \\ D\end{array}\right)$, where $B \in$ $\mathbb{R}^{m_{1} \times m_{1}}, C \in \mathbb{R}^{m_{2} \times m_{2}}, D \in \mathbb{R}^{m_{2} \times m_{1}}, m_{1}, m_{2} \in[1 ; n]$ and $m_{1}+m_{2}=n+1$. This fact may be seen by using [21, Th. 2.2.7] to establish the equivalence of the definition of irreducibility in Section II and [21, Def. 2.1.2] for $n \geq 2$. Next, as the last row of $\tilde{A}$ contains only nonzero entries, we conclude that the nonzero entries of $p$ are contained in a column of $C$. Hence, we may assume without loss of generality that the nonzero entries of $p$ are contained in the last column of $C$, i.e., that $T=\left(\begin{array}{cc}P & 0 \\ 0 & 1\end{array}\right)$, where $P \in \mathbb{R}^{n \times n}$ is a permutation matrix. Then, observe that the upper left $n \times n$ submatrix of $T A T^{\top}$ equals $P A P^{\top}$ and the first $m_{1}$ entries of the last column of $T \tilde{A} T^{\top}$ vanish. So, as $A$ is reducible, we may redefine $P$ to be such that $P A P^{\top}=\left(\begin{array}{ll}X & 0 \\ Z & Y\end{array}\right)$, which completes the proof.

Below, we denote $E$ by $E_{A, p}$ whenever clarity requires to specify $A \in \mathbb{R}_{+}^{n \times n}$ and $p \in \mathbb{R}_{+}^{n}$ in the definition (2) of $E$.

Proof of Theorem V.2. Sufficiency has already been established in the remark following the statement of Theorem V.1. To prove necessity, assume that both matrices in the statement are reducible. Note that $E_{A, p}(x)=E_{P A P^{\top}, P p}(P x)$ for any permutation matrix $P \in \mathbb{R}^{n \times n}$ and $x \in\left(\mathbb{R}_{+} \backslash\{0\}\right)^{n}$. Therefore, assume without loss of generality that $\left(\begin{array}{ll}A & p \\ 1 & 1\end{array}\right)$ is of the form (20). For $x \in \mathbb{R}^{n}$ let $x^{(1)}=\left(x_{1}, \ldots, x_{n_{1}}\right)$ and $x^{(2)}=\left(x_{n_{1}+1}, \ldots, x_{n}\right)$, i.e., $x=\left(x^{(1)}, x^{(2)}\right)$. Then

$$
E(x)=\prod_{i=1}^{n_{1}}\left(X x^{(1)}\right)_{i} \prod_{i=1}^{n_{2}}\left(Z x^{(1)}+Y x^{(2)}+\bar{p}\right)_{i}
$$

for any $x \in\left(\mathbb{R}_{+} \backslash\{0\}\right)^{n}$. Now, assume $x$ is a solution of $(12)$ and set $\xi_{\lambda}=\left(\lambda^{-n_{2} / n_{1}} x^{(1)}, \lambda x^{(2)}\right)$ for every $\lambda>0$. It follows that $\xi_{\lambda}$ is a feasible point of (12), for all $\lambda>0$. Next, if $Z x^{(1)} \neq 0$ or $\bar{p} \neq 0$ we obtain using (21) that

$$
E(x)>E_{X, 0}\left(x^{(1)}\right) \cdot E_{Y, 0}\left(x^{(2)}\right) .
$$

However, $E\left(\xi_{\lambda}\right)$ converges to the right hand side of (22) as $\lambda \rightarrow \infty$, which contradicts the choice of $x$. If $Z x^{(1)}=0$ and $\bar{p}=0$ then $E(x)=E\left(\xi_{\lambda}\right)$ for any $\lambda$, so any $\xi_{\lambda}$ is a solution of (12) for any $\lambda>0$.

Finally, we consider the general situation in Theorems III.5 and IV.1, where the growth bounds depend on the cell and the input symbol. In this case, the computation of an abstraction with finite $\bar{X}_{2}$ and finite $U_{2}$ requires a sequence of growth bounds $\left(\beta_{j}\right)_{j \in J}$ indexed by some finite set $J$. In particular, for any $i=(j, u) \in J \times U_{2}$ there are an essentially nonnegative matrix $L^{(i)} \in \mathbb{R}^{n \times n}$ and $v^{(i)} \in \mathbb{R}_{+}^{n}$ such that $\beta_{j}(r, u)$ is given by the right hand side of $(7)$ with $L^{(i)}$ and $v^{(i)}$ in place of $L(u)$ and $v(u)$, respectively, for all $r \in \mathbb{R}_{+}^{n}$. Therefore, we obtain by generalizing (12) the following heuristic to reduce the size of abstractions that have cells of volume $\exp (\gamma), \gamma \in \mathbb{R}$ : Pick the grid parameter to solve

$$
\min _{\xi>0} \widetilde{E}(\xi) \text { subject to } \exp (\gamma)=\prod_{i=1}^{n} \xi_{i},
$$

where $\widetilde{E}:\left(\mathbb{R}_{+} \backslash\{0\}\right)^{n} \rightarrow \mathbb{R}_{+}$is given by

$$
\widetilde{E}(\xi)=\sum_{i \in I} E_{A^{(i)}, p^{(i)}}(\xi) .
$$


Here, $A^{(i)}$ and $p^{(i)}$ are defined analogously to $A$ and $p$ in $(10)$, i.e., $A^{(i)}=\mathrm{id}+\exp \left(L^{(i)} \tau\right), p^{(i)}=$ $2\left(A^{(i)} z+v^{(i)}\right)$.

Theorem V.4 below provides sufficient conditions for (23) to possess a unique solution. To facilitate the practical verification of the conditions we will formulate them in terms of $L^{(i)}$ and $v^{(i)}$ rather than in terms of $A^{(i)}$ and $p^{(i)}$. Moreover, Theorem V.4 will imply that (23) can be solved numerically by solving (13) with $\widetilde{g}(x):=\widetilde{E}(\exp (x))$ in place of $g(x)$.

V.4 Theorem. Let $\gamma \in \mathbb{R}, \tau>0, z \in \mathbb{R}_{+}^{n}$, and let $I$ be a finite set. For every $i \in I$ define $A^{(i)}=$ $\mathrm{id}+\exp \left(L^{(i)} \tau\right), p^{(i)}=2\left(A^{(i)} z+v^{(i)}\right)$, where $L^{(i)} \in \mathbb{R}^{n \times n}$ is essentially nonnegative, and $v^{(i)} \in \mathbb{R}_{+}^{n}$. Let $V, \widetilde{E}$ and $\widetilde{g}$ be defined as in (14), (24) and above. For some $i \in I$ suppose that $L^{(i)}$ or

$$
\left(\begin{array}{cc}
L^{(i)} & z+L^{(i)} z+v^{(i)} \\
\mathbf{1} & 1
\end{array}\right)
$$

is irreducible. Then (23) possesses a unique solution. Moreover, $\widetilde{g}^{\prime \prime}(x) h^{2}>0$ for all $x \in V_{\gamma}$ and $h \in V_{0} \backslash\{0\}$, and $\widetilde{g}(x) \rightarrow \infty$ as $\|x\|_{\infty} \rightarrow \infty, x \in V_{\gamma}$.

The proof of Theorem V.4 requires the result below.

V.5 Lemma. Let $p \in \mathbb{R}_{+}^{n}$ and let $L \in \mathbb{R}^{n \times n}$ be essentially nonnegative. We have the following:

(i) $L$ is irreducible iff $\exp (L t)$ is irreducible for every $t>0$.

(ii) If $L$ is reducible, then $\left(\begin{array}{ll}L & p \\ \mathbf{1} & 1\end{array}\right)$ is irreducible iff $\left(\begin{array}{cc}\exp (L t) & p \\ \mathbf{1} & 1\end{array}\right)$ is irreducible for every $t>0$.

Proof. Necessity in (i) follows from [22, Rem. I.7.9] and [21, Th. 2.2.7] by establishing the equivalence between the definition of irreducibility in [22, Sec. I.7.4] and ours. Sufficiency in (i) follows from an evaluation of the exponential series.

To verify the necessary condition in (ii), first note that

$$
\left(L_{i, j} \neq 0, t>0\right) \Rightarrow \exp (L t)_{i, j} \geq 0
$$

if $L$ is nonnegative. To see that (26) also holds for essentially nonnegative matrices, choose $c>0$ such that $L+c$ id is nonnegative. Then $(26)$ holds since $\exp (L t)=\exp ((L+c$ id $) t) \exp (-c t)$. Thus, $A=\left(\begin{array}{cc}\exp (L t) & p \\ \mathbf{1} & 1\end{array}\right)$ arises from $B=\left(\begin{array}{cc}L t & p \\ 1 & 1\end{array}\right)$ by adding nonnegative values to the entries of $B$. Consequently, the irreducibility of $B$ passes over to $A$.

To prove sufficiency, assume that both $L$ and $\tilde{L}=\left(\begin{array}{ll}L & p \\ 1 & 1\end{array}\right)$ are reducible. By Lemma V.3 there exist permutation matrices $P$ and $\tilde{P}$ satisfying the following: $\tilde{P} \tilde{L} \tilde{P}^{\top}$ is of the form $(20)$ and $P \exp (L) P^{\top}=$ $\exp \left(P L P^{\top}\right)=\left(\begin{array}{cc}X^{\prime} & 0 \\ Z^{\prime} & Y^{\prime}\end{array}\right)$, where $X^{\prime}, Y^{\prime}, Z^{\prime}$ are matrices of the same dimensions as $X, Y, Z$ in $(20)$, respectively. Hence, $(\exp (L) p)$ is reducible by $[21$, Th. 2.2.7] as it can be transformed by $\tilde{P}$ to the form (20) with $X^{\prime}, Y^{\prime}, Z^{\prime}$ in place of $X, Y, Z$, respectively.

Proof of Theorem V.4. As (26) holds for $L^{(i)}$ in place of $L$, we may prove the theorem assuming $\exp \left(L^{(i)} \tau\right) z$ in place of $L^{(i)} z$ in $(25)$. Thus, by Lemma V.5 one of the matrices in the statement of Theorem V.1 with $A^{(i)}, p^{(i)}$ in place of $A, p$, respectively, is irreducible. Moreover, $A^{(i)}$ has positive diagonal [22, Th. I.7.4]. Now apply Theorem V.1 to $A^{(i)}$ and $p^{(i)}$ in place of $A$ and $p$ to see that $a(x):=$ $E_{A^{(i)}, p^{(i)}}(\exp (x))$ satisfies $a^{\prime \prime}(x) h^{2}>0$ for all $x \in V_{\gamma}$ and $h \in V_{0} \backslash\{0\}$, and $a(x) \rightarrow \infty$ as $\|x\|_{\infty} \rightarrow \infty, x \in$ $V_{\gamma}$. Moreover, every summand in $\widetilde{g}$ is convex and positive. So, as $a$ is a summand in $\widetilde{g}$ the assertions on $\widetilde{g}$ hold. Hence, (13) with $\widetilde{g}$ in place of $g$ possesses a unique solution [18, 4.3.3], and thus, so does (23).

\section{NUMERICAL EXAMPLE}

To demonstrate the benefits of the presented results, we consider the control system of a double pendulum that is mounted on a cart as investigated in [23]. The dynamics of this system can be decomposed into the motion of the two poles and the motion of the cart, which are coupled by the acceleration of the cart. Here, we consider only the motion of the poles. Specifically, we consider the 


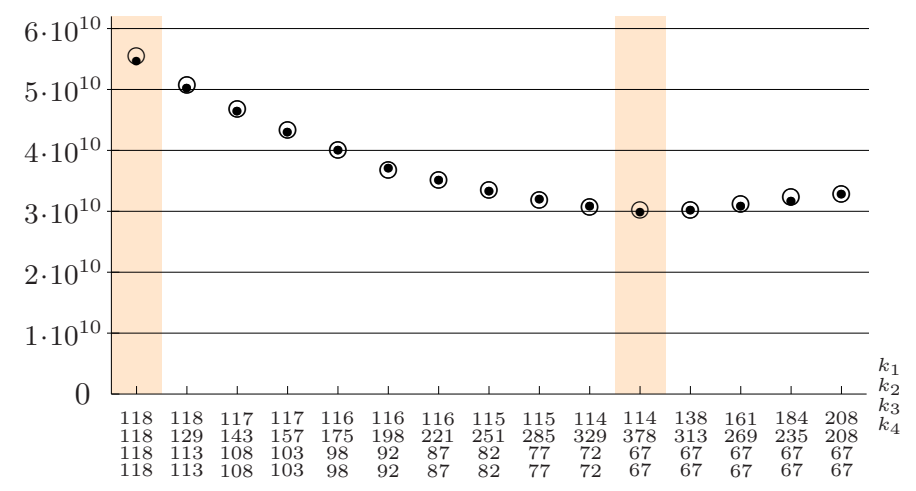

Figure 1. Predicted $(\bullet)$ and actual (o) number of transitions in abstractions based on the grid parameters $\left((\pi / 2+0.1) / k_{1}, 2 \pi / k_{2}, 11.4 / k_{3}, 11.4 / k_{4}\right)$ for the system $S_{1}$ considered in Section VI.

equations of motion given in [23, Tab. 2], rewritten as a first order system (3) with $n=4, \bar{U}=\mathbb{R}$, and $x=\left(\phi_{1}, \phi_{2}, \dot{\phi}_{1}, \dot{\phi}_{2}\right)$ in the notation of [23]. Specifically, $x_{1}$ and $x_{2}$ denotes the angle formed by the inner and outer, respectively, pole and the vertical ray, and $x_{3}$ and $x_{4}$ denote the corresponding angular velocities. The control input $u$ is the acceleration of the cart. See also [23, Fig. 1]. We additionally model uncertainties in friction forces in the links by virtue of $w=(0,0,0.018,0.028)$ in $(3)$.

We aim at steering the state of the system from the stable equilibrium point $(\pi, \pi, 0,0)$ to an ellipsoid centered at the lower unstable equilibrium point $x_{0}=(\pi, 0,0,0)$ given by $\left\{x \in \mathbb{R}^{4} \mid\left(x-x_{0}\right)^{\top} V\left(x-x_{0}\right) \leq 1\right\}$ where

$$
V=\left(\begin{array}{cccc}
0.247 & 0.153 & -0.023 & -0.026 \\
0.153 & 0.106 & 0.026 & -0.023 \\
-0.023 & 0.026 & 8.24 & 3.893 \\
-0.026 & -0.023 & 3.893 & 1.922
\end{array}\right)
$$

We assume measurement errors (4) with $z=(b, b, 2 b, 2 b), b=2 \pi / 2^{14}$ which are motivated by 14 -bit quantized measurements of the angles. Additionally, we require the state $x \in \mathbb{R}^{4}$ of the system not to leave $\bar{X}=[\pi / 2, \pi+0.1] \times[0,2 \pi] \times[-5.7,5.7] \times[-5.7,5.7]$ and we identify $x$ and $x+(0,2 \pi k, 0,0)$ for any $k \in \mathbb{Z}$. The latter means that we do not impose restrictions on the outer angle.

We shall solve this control task for the sampled system $S_{1}$ associated with (3) and sampling time $\tau=0.01$ using the synthesis procedure outlined in Sections I and III, in which we focus on the computation of abstractions. Using Theorem III.5, we will compute two abstractions $S_{2}=\left(X_{2}, U_{2}, F_{2}\right)$ and $S_{2}^{\prime}=\left(X_{2}^{\prime}, U_{2}^{\prime}, F_{2}^{\prime}\right)$ for $S_{1}$, where $S_{2}$ is based on a naive choice of the grid parameter, and the grid parameter for $S_{2}^{\prime}$ is chosen using the results in Section V.

We begin with the details to $S_{2}$. We let $U_{2}$ consist of 5 elements equally spaced on $9.81 \cdot[-3.5,3.5]$. Next, we let $\bar{X}_{2}$ in Theorem III.5 be a cover of $\bar{X}$ and let $X_{2}$ be a grid of the form (5) with grid parameter

$$
\eta=\left(\frac{\pi / 2+0.1}{118}, \frac{2 \pi}{118}, \frac{11.4}{118}, \frac{11.4}{118}\right) \approx(0.014,0.053,0.097,0.097)
$$

$\eta$ is a naive choice as each component of $\bar{X}$ is subdivided into 118 intervals of equal length. Then $\bar{X}_{2}$ consists of about $194 \cdot 10^{6}$ cells. The transition function $F_{2}$ is computed according to Theorem III.5, where the required growth bounds are obtained by methods presented in [1]. The computation of $S_{2}$ requires $206 \mathrm{~GB}$ RAM and $2.5 \mathrm{~h}$ cpu time. $S_{2}$ contains about $55.7 \cdot 10^{9}$ transitions and $54.6 \cdot 10^{9}$ transitions have been predicted by means of (24). All computations in this section are run on a single thread of an Intel Xeon E5-2687W $(3.1 \mathrm{GHz})$.

In contrast, for $S_{2}^{\prime}$ we let $U_{2}^{\prime}=U_{2}$ but choose the grid parameter as the solution of (23) under the constraint that the cells of $X_{2}^{\prime}$ have the same volume as those of $X_{2}$ and the additional constraints $\xi_{i} \leq 0.17, i \in[1 ; 4]$,

$$
\eta^{\prime}=\left(\frac{\pi / 2+0.1}{114}, \frac{2 \pi}{378}, \frac{11.4}{67}, \frac{11.4}{67}\right) \approx(0.015,0.017,0.17,0.17)
$$


and define $X_{2}^{\prime}$ in the same way as $X_{2}$, with $\eta^{\prime}$ in place of $\eta$. The computation of $S_{2}^{\prime}$ requires $134 \mathrm{~GB}$ RAM and 73 min cpu time. $S_{2}^{\prime}$ contains about $30.3 \cdot 10^{9}$ transitions and the prediction has been $30.0 \cdot 10^{9}$ transitions.

In summary, the number of transitions, computational time and memory consumption is reduced by $46 \%, 51 \%$ and $35 \%$, respectively, compared to a naive choice of the aspect ratio. Moreover, in contrast to the auxiliary control problem for $S_{2}$, the one for $S_{2}^{\prime}$ is solvable, due to a reduced number of spurious transitions.

The success of our method to reduce the size of abstractions depends to a great extend on the accuracy by which the functional in (23) predicts the number of transitions. That accuracy is illustrated in Fig. 1 for a number of additional abstractions for $S_{1}$ with varying grid parameters. It turns out that the prediction possesses an error of less than $2 \%$.

\section{REFERENCES}

[1] G. Reissig, A. Weber, and M. Rungger, "Feedback refinement relations for the synthesis of symbolic controllers," IEEE Trans. Automat. Control, vol. 62, no. 4, pp. 1781-1796, Apr. 2017, DOI:10.1109/TAC.2016.2593947, arXiv:1503.03715.

[2] P. Tabuada, Verification and control of hybrid systems. New York: Springer, 2009.

[3] M. Rungger and O. Stursberg, "On-the-fly model abstraction for controller synthesis," in American Control Conference (ACC), 2012, pp. 2645-2650.

[4] M. Rungger, M. Mazo, and P. Tabuada, "Specification-guided controller synthesis for linear systems and safe linear-time temporal logic," in Proc. 16th Intl. Conf. Hybrid Systems: Computation and Control (HSCC), Philadelphia, PA, U.S.A., Apr. 8-11, 2013. ACM, 2013, pp. 333-342.

[5] G. Pola, A. Borri, and M. D. Di Benedetto, "Integrated design of symbolic controllers for nonlinear systems," IEEE Trans. Automat. Control, vol. 57, no. 2, pp. 534-539, 2012.

[6] A. Girard, G. Gössler, and S. Mouelhi, "Safety controller synthesis for incrementally stable switched systems using multiscale symbolic models," IEEE Transactions on Automatic Control, vol. 61, no. 6, pp. 1537-1549, June 2016.

[7] Y. Tazaki and J. Imura, "Discrete-state abstractions of nonlinear systems using multi-resolution quantizer," in Proc. 12th Intl. Conf. Hybrid Systems: Computation and Control (HSCC), San Francisco, U.S.A., Apr. 13-15, 2009, ser. Lect. Notes Computer Science, R. Majumdar and P. Tabuada, Eds., vol. 5469. Springer, 2009, pp. 351-365.

[8] S. Mouelhi, A. Girard, and G. Gössler, "Cosyma: A tool for controller synthesis using multi-scale abstractions," in Proc. 16th Intl. Conf. Hybrid Systems: Computation and Control (HSCC), Philadelphia, PA, U.S.A., Apr. 8-11, 2013. New York, NY, USA: ACM, 2013, pp. 83-88.

[9] E. Le Corronc, A. Girard, and G. Goessler, "Mode sequences as symbolic states in abstractions of incrementally stable switched systems," in Proc. 52th IEEE Conf. Decision and Control (CDC), Florence, Italy, 10-13 Dec. $2013 . \quad$ New York: IEEE, 2013, pp. 3225-3230.

[10] M. Zamani, I. Tkachev, and A. Abate, "Bisimilar symbolic models for stochastic control systems without state-space discretization," in Proc. 17th Intl. Conf. Hybrid Systems: Computation and Control (HSCC), Berlin, Germany, Apr. 15-17, 2014. New York, NY, USA: ACM, 2014, pp. 41-50.

[11] G. Reissig and M. Rungger, "Feedback refinement relations for symbolic controller synthesis," in Proc. IEEE Conf. Decision and Control (CDC), Los Angeles, CA, U.S.A., 15-17 Dec. 2014. New York: IEEE, 2014, pp. 88-94.

[12] D. Ž. Djoković, "Note on nonnegative matrices," Proc. Amer. Math. Soc., vol. 25, pp. 80-82, 1970.

[13] D. London, "On matrices with a doubly stochastic pattern," J. Math. Anal. Appl., vol. 34, pp. 648-652, 1971.

[14] M. Rungger, A. Weber, and G. Reissig, "State space grids for low complexity abstractions," in Proc. IEEE Conf. Decision and Control (CDC), Osaka, Japan, 15-18 Dec. 2015. New York: IEEE, 2015, pp. 6139-6146.

[15] R. T. Rockafellar and R. J.-B. Wets, Variational analysis, ser. Grundlehren der Mathematischen Wissenschaften. Berlin: Springer-Verlag, 1998, vol. 317, 3rd corr printing 2009.

[16] A. F. Filippov, Differential equations with discontinuous righthand sides, ser. Mathematics and its Applications (Soviet Series). Kluwer Academic Publishers Group, Dordrecht, 1988, vol. 18, translated from the Russian.

[17] G. Reißig, "Computing abstractions of nonlinear systems," IEEE Trans. Automat. Control, vol. 56, no. 11, pp. 2583-2598, Nov. 2011.

[18] J. M. Ortega and W. C. Rheinboldt, Iterative solution of nonlinear equations in several variables, ser. Classics in Applied Mathematics. Philadelphia, PA: Society for Industrial and Applied Mathematics (SIAM), 2000, vol. 30, reprint of the 1970 original.

[19] M. V. Solodov, "Global convergence of an SQP method without boundedness assumptions on any of the iterative sequences," Math. Programming, vol. 118, no. 1, Ser. A, pp. 1-12, 2009.

[20] R. Tarjan, "Depth first search and linear graph algorithms," SIAM Journal on Computing, 1972.

[21] A. Berman and R. J. Plemmons, Nonnegative matrices in the mathematical sciences, ser. Classics in Applied Mathematics. Philadelphia, PA: Society for Industrial and Applied Mathematics (SIAM), 1994, vol. 9, revised reprint of the 1979 original.

[22] T. Kato, A short introduction to perturbation theory for linear operators. New York: Springer-Verlag, 1982.

[23] K. Graichen, M. Treuer, and M. Zeitz, "Swing-up of the double pendulum on a cart by feedforward and feedback control with experimental validation," Automatica J. IFAC, vol. 43, no. 1, pp. 63-71, 2007. 